\title{
On the length of chains in a metric space.
}

\author{
Mathav Murugan*
}

May 12, 2020

\begin{abstract}
We obtain an upper bound on the minimal number of points in an $\varepsilon$-chain joining two points in a metric space. This generalizes a bound due to Hambly and Kumagai (1999) for the case of resistance metric on certain self-similar fractals. As an application, we deduce a condition on $\varepsilon$-chains introduced by Grigor'yan and Telcs (2012). This allows us to obtain sharp bounds on the heat kernel for spaces satisfying the parabolic Harnack inequality without assuming further conditions on the metric. A snowflake transform on the Euclidean space shows that our bound is sharp.
\end{abstract}

\section{Introduction}

The fundamental solution of the heat equation (or heat kernel) on $\mathbb{R}^{n}$ is given by the Gauss Weierstrass kernel

$$
p_{t}(x, y)=\frac{1}{(4 \pi t)^{n / 2}} \exp \left(-\frac{d(x, y)^{2}}{4 t}\right), \quad \text { for all } x, y \in \mathbb{R}^{n}, t>0 .
$$

From a probabilistic viewpoint, the heat kernel can be interpreted as the transition probability density of the diffusion generated by the Laplacian $\Delta$. More generally, for any uniformly elliptic, divergence form operator $\mathcal{L} u=\sum_{i, j=0}^{n} \frac{\partial}{\partial x_{i}}\left(a_{i j}(x) \frac{\partial u}{\partial x_{j}}\right)$ on $\mathbb{R}^{n}$, Aronson [Ar67] proved that the heat kernel $p_{t}(x, y)$ of the corresponding heat equation $\partial_{t} u-\mathcal{L} u=0$ satisfies

$$
\frac{c_{1}}{V\left(x, t^{1 / 2}\right)} \exp \left(-\frac{d(x, y)^{2}}{c_{1} t}\right) \leq p_{t}(x, y) \leq \frac{C_{1}}{V\left(x, t^{1 / 2}\right)} \exp \left(-\frac{d(x, y)^{2}}{C_{1} t}\right)
$$

for all $x, y \in \mathbb{R}^{n}, t>0$, where $c_{1}, C_{1} \in(0, \infty)$. Here $V(x, r)$ denotes the Lebesgue measure of the Euclidean ball $B(x, r)$ centered at $x$ with radius $r$.

${ }^{*}$ Research partially supported by NSERC (Canada). 
To prove the above lower bound on $p_{t}(x, y)$, the first step is to obtain the lower bound under the additional restriction that $d(x, y) \leq C t^{1 / 2}$. Such an estimate is called a near diagonal lower bound. In order to obtain the full lower bound from a near diagonal lower bound, one chooses a sequence of points (called a chain) $\left\{x_{i}\right\}_{i=0}^{n}$ such that $x_{0}=x, x_{n}=y$ and $n \in \mathbb{N}$ such that $d\left(x_{i}, x_{i+1}\right) \leq C(t / n)^{1 / 2} / 2$ for all $i=0,1, \ldots, n-1$. Then we use Chapman-Kolmogorov equation to obtain the estimate

$$
p_{t}(x, y) \geq \int_{B\left(x_{n-1}, C(t / n)^{1 / 2} / 2\right)} \cdots \int_{B\left(x_{1}, C(t / n)^{1 / 2} / 2\right)} \prod_{i=0}^{n-1} p_{t / n}\left(y_{i}, y_{i+1}\right) d y_{1} \ldots d y_{n-1}
$$

where $y_{0}=x, y_{n}=y$. By optimizing over $n$ and the sequence $\left\{x_{i}\right\}_{i=0}^{n}$ and using the near diagonal lower bound, we obtain the full lower bound on the heat kernel $p_{t}(x, y)$. This method of obtaining full heat kernel lower bound is called the chaining argument.

The use of chaining argument to obtain heat kernel estimates is classical [AS67, Ar67]. Such chaining arguments are also used to obtain heat kernel estimates on fractals; see [Bar98] for a general introduction to diffusions on fractals. In this work, we address the natural converse question: Do heat kernel estimates imply the existence of short chains? Our main result provides an upper bound on the length of chains, which in some sense is the best possible. The goal of this work is to obtain sharp quantitative bounds on the connectivity of a metric space. This will be expressed as bounds on the length of chains.

\subsection{Framework and definitions}

We recall the definition of a chain in a metric space $(X, d)$.

Definition 1.1. We say that a sequence $\left\{x_{i}\right\}_{i=0}^{N}$ of points in $X$ is an $\varepsilon$-chain between points $x, y \in X$ if

$$
x_{0}=x, \quad x_{N}=y, \quad \text { and } \quad d\left(x_{i}, x_{i+1}\right)<\varepsilon \quad \text { for all } i=0,1, \ldots, N-1 .
$$

For any $\varepsilon>0$ and $x, y \in X$, define

$$
d_{\varepsilon}(x, y)=\inf _{\left\{x_{i}\right\} \text { is } \varepsilon \text {-chain }} \sum_{i=0}^{N-1} d\left(x_{i}, x_{i+1}\right)
$$

where the infimum is taken over all $\varepsilon$-chains $\left\{x_{i}\right\}_{i=0}^{N}$ between $x, y$ with arbitrary $N$. Note that if $(X, d)$ is a geodesic space, then $d_{\varepsilon}(x, y)=d(x, y)$ for all $\varepsilon>0, x, y \in X$.

Throughout this paper, we consider a complete, locally compact separable metric space $(X, d)$, equipped with a Radon measure $m$ with full support, i.e., a Borel measure $m$ on $X$ which is finite on any compact set and strictly positive on any non-empty open set. Such a triple $(X, d, m)$ is referred to as a metric measure space. Then we set $\operatorname{diam}(X, d):=$ $\sup _{x, y \in X} d(x, y)$ and $B(x, r):=\{y \in X \mid d(x, y)<r\}$ for $x \in X$ and $r>0$.

Let $(\mathcal{E}, \mathcal{F})$ be a symmetric Dirichlet form on $L^{2}(X, m)$. In other words, the domain $\mathcal{F}$ is a dense linear subspace of $L^{2}(X, m)$, such that $\mathcal{E}: \mathcal{F} \times \mathcal{F} \rightarrow \mathbb{R}$ is a non-negative 
definite symmetric bilinear form which is closed $(\mathcal{F}$ is a Hilbert space under the inner product $\left.\mathcal{E}_{1}(\cdot, \cdot):=\mathcal{E}(\cdot, \cdot)+\langle\cdot, \cdot\rangle_{L^{2}(X, m)}\right)$ and Markovian (the unit contraction operates on $\mathcal{F} ;(u \vee 0) \wedge 1 \in \mathcal{F}$ and $\mathcal{E}((u \vee 0) \wedge 1,(u \vee 0) \wedge 1) \leq \mathcal{E}(u, u)$ for any $u \in \mathcal{F})$. Recall that $(\mathcal{E}, \mathcal{F})$ is called regular if $\mathcal{F} \cap \mathcal{C}_{\mathrm{c}}(X)$ is dense both in $\left(\mathcal{F}, \mathcal{E}_{1}\right)$ and in $\left(\mathcal{C}_{\mathrm{c}}(X),\|\cdot\|_{\text {sup }}\right)$. Here $\mathcal{C}_{\mathrm{c}}(X)$ is the space of $\mathbb{R}$-valued continuous functions on $X$ with compact support.

For a function $u \in \mathcal{F}$, let $\operatorname{supp}_{m}[u]$ denote the support of the measure $|u| d m$, i.e., the smallest closed subset $F$ of $X$ with $\int_{X \backslash F}|u| d m=0$; note that $\operatorname{supp}_{m}[u]$ coincides with the closure of $X \backslash u^{-1}(\{0\})$ in $X$ if $u$ is continuous. Recall that $(\mathcal{E}, \mathcal{F})$ is called strongly local if $\mathcal{E}(u, v)=0$ for any $u, v \in \mathcal{F}$ with $\operatorname{supp}_{m}[u], \operatorname{supp}_{m}[v]$ compact and $v$ is constant $m$-almost everywhere in a neighborhood of $\operatorname{supp}_{m}[u]$. The pair $(X, d, m, \mathcal{E}, \mathcal{F})$ of a metric measure space $(X, d, m)$ and a strongly local, regular symmetric Dirichlet form $(\mathcal{E}, \mathcal{F})$ on $L^{2}(X, m)$ is termed a metric measure Dirichlet space, or an MMD space. We refer to [FOT, CF] for a comprehensive account of the theory of symmetric Dirichlet forms.

We recall the definition of energy measures associated to an MMD space. Note that $f g \in \mathcal{F}$ for any $f, g \in \mathcal{F} \cap L^{\infty}(X, m)$ by [FOT, Theorem 1.4.2-(ii)] and that $\{(-n) \vee$ $(f \wedge n)\}_{n=1}^{\infty} \subset \mathcal{F}$ and $\lim _{n \rightarrow \infty}(-n) \vee(f \wedge n)=f$ in norm in $\left(\mathcal{F}, \mathcal{E}_{1}\right)$ by [FOT, Theorem 1.4.2-(iii)].

Definition 1.2 (cf. [FOT, (3.2.14) and (3.2.15)]). Let $(X, d, m, \mathcal{E}, \mathcal{F})$ be an MMD space. The energy measure $\Gamma(f, f)$ of $f \in \mathcal{F}$ is defined, first for $f \in \mathcal{F} \cap L^{\infty}(X, m)$ as the unique $([0, \infty]$-valued) Borel measure on $X$ with the property that

$$
\int_{X} g d \Gamma(f, f)=\mathcal{E}(f, f g)-\frac{1}{2} \mathcal{E}\left(f^{2}, g\right) \quad \text { for all } g \in \mathcal{F} \cap \mathcal{C}_{\mathrm{c}}(X),
$$

and then by $\Gamma(f, f)(A):=\lim _{n \rightarrow \infty} \Gamma((-n) \vee(f \wedge n),(-n) \vee(f \wedge n))(A)$ for each Borel subset $A$ of $K$ for general $f \in \mathcal{F}$.

The notion of energy measure can be extended to the local Dirichlet space $\mathcal{F}_{\text {loc }}$, which is defined as

$$
\mathcal{F}_{\text {loc }}:=\left\{\begin{array}{l|l}
f \in L_{\text {loc }}^{2}(X, m) & \begin{array}{l}
\text { For any relatively compact open subset } V \text { of } X, \\
\text { there exists } f^{\#} \in \mathcal{F} \text { such that } f \mathbb{1}_{V}=f^{\#} \mathbb{1}_{V} m \text {-a.e. }
\end{array}
\end{array}\right\} .
$$

For any $f \in \mathcal{F}_{\text {loc }}$ and for any relatively compact open set $V \subset X$, we define

$$
\Gamma(f, f)(V)=\Gamma\left(f^{\#}, f^{\#}\right)(V),
$$

where $f^{\#}$ is as in the definition of $\mathcal{F}_{\text {loc }}$. Since $(\mathcal{E}, \mathcal{F})$ is strongly local, the value of $\Gamma\left(f^{\#}, f^{\#}\right)(V)$ does not depend on the choice of $f^{\#}$, and is therefore well defined. Since $X$ is locally compact, this defines a Radon measure $\Gamma(f, f)$ on $X$.

Definition 1.3 (Capacity between sets). For subsets $A, B \subset X$, we define $\mathcal{F}(A, B):=\{f \in \mathcal{F}: f \equiv 1$ on a neighborhood of $A$ and $f \equiv 0$ on a neighborhood of $B\}$, and the capacity $\operatorname{Cap}(A, B)$ as

$$
\operatorname{Cap}(A, B)=\inf \{\mathcal{E}(f, f): f \in \mathcal{F}(A, B)\} .
$$


The main result of this work provides upper bounds on $d_{\varepsilon}(x, y)$ based on analytic conditions on an MMD space that we introduce now. Henceforth, we fix a continuous increasing bijection $\Psi:(0, \infty) \rightarrow(0, \infty)$ such that for all $0<r \leq R$,

$$
C^{-1}\left(\frac{R}{r}\right)^{\beta_{1}} \leq \frac{\Psi(R)}{\Psi(r)} \leq C\left(\frac{R}{r}\right)^{\beta_{2}}
$$

for some constants $0<\beta_{1}<\beta_{2}$ and $C>1$. Throughout this work, the function $\Psi$ is meant to denote the space time scaling of the process.

Definition 1.4. We recall the following properties that an MMD space $(X, d, m, \mathcal{E}, \mathcal{F})$ may satisfy:

We say that $(X, d, m)$ satisfies the volume doubling property (VD) if there exists $C_{D} \geq 1$ such that

$$
m(B(x, 2 r)) \leq C_{D} m(B(x, r)), \quad \text { for all } x \in X, r>0 .
$$

We say that $(X, d, m, \mathcal{E}, \mathcal{F})$ satisfies the Poincaré inequality $\operatorname{PI}(\Psi)$, if there exist constants $C, A \geq 1$ such that for all $x \in X, r \in(0, \infty)$ and $f \in \mathcal{F}$

$$
\int_{B(x, R)}(f-\bar{f})^{2} d m \leq C \Psi(r) \int_{B(x, A r)} d \Gamma(f, f),
$$

where $\bar{f}=m(B(x, r))^{-1} \int_{B(x, r)} f d \mu$. If all balls are relatively compact, then the above inequality can be extended to all $f \in \mathcal{F}_{\text {loc }}$.

We say that $(X, d, m, \mathcal{E}, \mathcal{F})$ satisfies the capacity estimate $\operatorname{cap}(\Psi)_{\leq}$if there exist $C_{1}, A_{1}, A_{2}>1$ such that for all $x \in X, 0<R<\operatorname{diam}(X, d) / A_{2}$,

$$
\operatorname{Cap}\left(B(x, R), B\left(x, A_{1} R\right)^{c}\right) \leq C_{1} \frac{m(B(x, R))}{\Psi(R)} . \quad \operatorname{cap}(\Psi)_{\leq}
$$

We recall the definition of the heat kernel corresponding to an MMD space.

Definition 1.5. Let $(X, d, m, \mathcal{E}, \mathcal{F})$ be an MMD space, and let $\left\{P_{t}\right\}_{t>0}$ denote its associated Markov semigroup. A family $\left\{p_{t}\right\}_{t>0}$ of non-negative Borel measurable functions on $X \times X$ is called the heat kernel of $(X, d, m, \mathcal{E}, \mathcal{F})$, if $p_{t}$ is the integral kernel of the operator $P_{t}$ for any $t>0$, that is, for any $t>0$ and for any $f \in L^{2}(X, m)$,

$$
P_{t} f(x)=\int_{X} p_{t}(x, y) f(y) d m(y) \quad \text { for } m \text {-almost every } x \in X .
$$

We remark that not every MMD space $(X, d, m, \mathcal{E}, \mathcal{F})$ has a heat kernel. The existence of heat kernel is an issue in general.

\subsection{Main results}

Our main result is the following upper bound on $d_{\varepsilon}$. 
Theorem 1.6. Let $(X, d, m, \mathcal{E}, \mathcal{F})$ be an $M M D$ space that satisfies (VD), $\mathrm{PI}(\Psi)$, and $\operatorname{cap}(\Psi)_{\leq}$, where $\Psi$ satisfies (1.3). Then there exists $C>1$ such that for all $\varepsilon>0$ and for all $x, y \in X$ that satisfy $d(x, y) \geq \varepsilon$, we have

$$
\frac{d_{\varepsilon}(x, y)^{2}}{\varepsilon^{2}} \leq C \frac{\Psi(d(x, y))}{\Psi(\varepsilon)}
$$

In particular, for all $x, y \in X$, we have

$$
\lim _{\varepsilon \rightarrow 0} \Psi(\varepsilon) \frac{d_{\varepsilon}(x, y)}{\varepsilon}=0
$$

Remark 1.7. (a) If $\Psi(r)=r^{2},(1.4)$ implies the chain condition $d_{\varepsilon}(x, y) \lesssim d(x, y)$ for all $\varepsilon>0, x, y \in X$.

(b) If $\Psi(r)=r^{\beta}$, then (1.4) and the triangle inequality $d_{\varepsilon}(x, y) \geq d(x, y)$ imply that

$$
\frac{d(x, y)^{2}}{\varepsilon^{2}} \leq \frac{d_{\varepsilon}(x, y)^{2}}{\varepsilon^{2}} \leq C \frac{d(x, y)^{\beta}}{\varepsilon^{\beta}}
$$

for all $x, y \in X, \varepsilon>0$ with $d(x, y) \geq \varepsilon$. By letting $\varepsilon \rightarrow 0$, we give a new proof of the known fact that $\beta \geq 2$ must necessarily hold.

(c) Let $\Psi(r)=r^{\beta}$ with $\beta \geq 2$. Consider the Dirichlet form corresponding to the Brownian motion on $\mathbb{R}^{d}$ with Lebesgue measure as the symmetric measure, and the snowflake metric $d(x, y)=\|x-y\|_{2}^{2 / \beta}$, where $\|x-y\|_{2}$ denotes the Euclidean distance (cf. [MT, Defintion 1.2.8] for the terminology 'snowflake metric'). In this case, it is easy to obtain

$$
\Psi(\varepsilon) \frac{d_{\varepsilon}(x, y)^{2}}{\varepsilon^{2}} \asymp \Psi(d(x, y)) \asymp\|x-y\|_{2}^{2}
$$

for all $x, y \in \mathbb{R}^{d}, \varepsilon>0$ with $\varepsilon<d(x, y)$. Hence, the bound (1.4) is sharp for all $\beta \geq 2$.

(d) Theorem 1.6 provides a new proof to an estimate due to Hambly and Kumagai [HaKu99, Lemma 3.3]. Based on the results in [HaKu99], the estimate (1.5) was introduced by Grigor'yan and Telcs in [GT12, (1.8)] to obtain sharp estimates of the heat kernel (cf. Corollary 2.9).

By [GT12, Theorem 6.5] along with (1.5), we have the following corollary (see Theorem 2.11 for generalization to arbitrary scale functions $\Psi$ ).

Corollary 1.8. Let $(X, d, m, \mathcal{E}, \mathcal{F})$ be an $M M D$ space that satisfies the following subGaussian estimate on its heat kernel $p_{t}(\cdot, \cdot)$ : there exists $\beta \geq 2, C, c>0$ such that

$\frac{c}{V\left(x, t^{1 / \beta}\right)} \exp \left(-\left(\frac{d(x, y)^{\beta}}{c t}\right)^{1 /(\beta-1)}\right) \leq p_{t}(x, y) \leq \frac{C}{V\left(x, t^{1 / \beta}\right)} \exp \left(-\left(\frac{d(x, y)^{\beta}}{C t}\right)^{1 /(\beta-1)}\right)$

for all $x, y \in X, t>0$, where $V(x, r)=m(B(x, r))$. Then the metric d satisfies the chain condition: there exists $K>1$ such that

$$
d(x, y) \leq d_{\varepsilon}(x, y) \leq K d(x, y) \quad \text { for all } \varepsilon>0 \text { and for all } x, y \in X .
$$


Remark 1.9. (a) The chain condition (1.7) admits the following characterization. Let $(X, d)$ be metric space such that the open balls $B(x, r)$ are relatively compact for all $x \in X, r>0$. Then $(X, d)$ satisfies the chain condition if and only if there exists a geodesic metric $\rho$ such that $d$ is bi-Lipschitz equivalent to $\rho$ [KM, Proposition A.1]. Recall that $(X, \rho)$ is geodesic, if for any two points $x, y \in X$, there exists a function $\gamma:[0, \rho(x, y)] \rightarrow X$ such that $\rho(\gamma(s), \gamma(t))=|s-t|$ for all $s, t \in[0, \rho(x, y)]$.

(b) Corollary 1.8 was previously known only for the case $\beta=2$. By a version of Varadhan's asymptotic formula in [HR, Theorem 1.1], we obtain that $d$ is bi-Lipschitz equivalent to the intrinsic metric. Hence by the remark above, $d$ satisfies the chain condition. However, the intrinsic metric vanishes identically for the case $\beta>2$. This suggests the need for a completely different approach when $\beta>2$.

(c) The chain condition plays an essential role in the proof of singularity of energy measures in $[\mathrm{KM}]$ for spaces satisfying the sub-Gaussian heat kernel estimate.

Recall that the parameter $\beta$ in Corollary 2.9 is called the walk dimension. The following result can be viewed as a generalization of the result that the walk dimension is at least two.

Corollary 1.10. Let $(X, d, m, \mathcal{E}, \mathcal{F})$ be an $M M D$ space that satisfies $(\mathrm{VD}), \mathrm{PI}(\Psi)$, and $\operatorname{cap}(\Psi)_{\leq}$, where $\Psi$ satisfies (1.3). Then there exists $C_{1} \geq 1$ such that

$$
\frac{\Psi(r)}{\Psi(s)} \geq C_{1}^{-1}\left(\frac{r}{s}\right)^{2}, \quad \text { for all } 0<s \leq r<\operatorname{diam}(X, d) .
$$

Notation. In the following, we will use the notation $A \lesssim B$ for quantities $A$ and $B$ to indicate the existence of an implicit constant $C \geq 1$ depending on some inessential parameters such that $A \leq C B$. We write $A \asymp B$, if $A \lesssim B$ and $B \lesssim A$.

\section{Proofs}

\subsection{Connectedness}

We recall the notion of a net in a metric space.

Definition 2.1. Let $(X, d)$ be a metric space and let $\varepsilon>0$. A maximal $\varepsilon$-separated subset $V \subset X$ is called an $\varepsilon$-net; in other words, $V$ satisfies the following properties:

(a) $V$ is $\varepsilon$-separated; that is, $d(x, y) \geq \varepsilon$ whenever $x, y \in V$ and $x \neq y$.

(b) (maximality) If $W \supseteq V$ and $W$ is $\varepsilon$-separated, then $W=V$.

As a first step towards (1.4), we show that $d_{\varepsilon}(x, y)$ is finite for all $\varepsilon>0, x, y \in X$. 
Lemma 2.2. Let $(X, d, m, \mathcal{E}, \mathcal{F})$ be an $M M D$ space that satisfies $(\mathrm{VD}), \operatorname{PI}(\Psi)$, where $\Psi$ satisfies (1.3). Then

$$
d_{\varepsilon}(x, y)<\infty \quad \text { for all } x, y \in X \text { and for all } \varepsilon>0 .
$$

Proof. Let $x \in X, \varepsilon>0$ and $U_{x}=\left\{y: d_{\varepsilon}(x, y)<\infty\right\}, V_{x}=X \backslash U_{x}$. Clearly $U_{x}$ is open, since $z \in U_{x}$ implies $B(z, \varepsilon) \subset U_{x}$. Similarly, $V_{x}$ is open since $z \in V_{x}$ implies $B(z, \varepsilon) \subset V_{x}$. The above statements also imply that

$$
\inf \left\{d(y, z): y \in U_{x}, z \in V_{x}\right\} \geq \varepsilon .
$$

Let $N$ denote a $\varepsilon / 2$-net in $(X, d)$. Let $\phi_{z} \in C_{c}(X) \cap \mathcal{F}$ such that $1 \geq \phi_{z} \geq 0,\left.\phi_{z}\right|_{B(z, \varepsilon / 2)} \equiv 1$, and $\operatorname{supp}\left(\phi_{z}\right) \subset B(z, \varepsilon)$. Define

$$
\phi(y)=\sup _{z \in N \cap U_{x}} \phi_{z}(y) \quad \text { for all } y \in X .
$$

By (2.1), and $\cup_{z \in N} B(z, \varepsilon / 2)=X$, we obtain

$$
\phi \equiv 1_{U_{x}}
$$

For any precompact open set $U$, we have $\left|\left\{z \in N \cap U_{x}: B(z, \varepsilon) \cap U\right\}\right|<\infty$. In this case, by setting $N_{U}=\left\{z \in N \cap U_{x}: B(z, \varepsilon) \cap U\right\}$, we have

$$
\inf _{z \in N_{U}} \phi_{z}(y)=\phi(y) \quad \text { for all } y \in U, \quad \inf _{z \in N_{U}} \phi_{z} \in \mathcal{F} \cap C(X) .
$$

Therefore $\phi=1_{U_{x}} \in \mathcal{F}_{\text {loc }} \cap C(X)$. By [CF, Theorem 4.3.8], the push-forward measure of $\Gamma(\phi, \phi)$ by $\phi$ is absolutely continuous with respect to the 1-dimensional Lebesgue measure. Since $\{0,1\}$ has zero Lebesgue measure, we obtain

$$
\Gamma(\phi, \phi)(B)=0
$$

for all balls $B=B(x, R), R>0$. Since $\phi$ is continuous, by $\operatorname{PI}(\Psi), \phi$ is constant on all balls $B(x, R)$. Therefore $\phi(y)=\phi(x)=1$ for all $y \in B(x, R), R>0$. Therefore $\phi \equiv 1$. Hence $V_{x}=\emptyset$. Since $x \in X, \varepsilon>0$ are arbitrary, we obtain the desired estimate.

Recall that a metric space $(X, d)$ is said to be uniformly perfect, if there exists $C>1$ such that $B(x, r) \backslash B(x, r / C) \neq \emptyset$ for all $x \in X, r>0$ that satisfy $B(x, r) \neq X$.

Corollary 2.3. Let $(X, d, m, \mathcal{E}, \mathcal{F})$ be an $M M D$ space that $(\mathrm{VD}), \mathrm{PI}(\Psi)$, where $\Psi$ satisfies (1.3). Then $(X, d)$ is a uniformly perfect metric space.

Proof. Let $B(x, r)$ be a ball such that $B(x, r) \neq X$. Let $y \in X \backslash B(x, r)$. By Lemma 2.2 , there exists an $\varepsilon$-chain $\left\{x_{i}\right\}_{i=0}^{N}, x_{0}=x, x_{N}=y$ between $x$ and $y$ for $\varepsilon=r / 4$. Since $d\left(x, x_{0}\right)=0, d\left(x, x_{N}\right) \geq r$ and $\left|d\left(x, x_{i}\right)-d\left(x, x_{i+1}\right)\right| \leq d\left(x_{i}, x_{i+1}\right) \leq r / 4$ for all $i=0, \ldots, N-1$, we have $d\left(x, x_{j}\right) \in[r / 2, r)$ for some $1 \leq j \leq N-1$. Hence

$$
B(x, r) \backslash B(x, r / 2) \neq \emptyset .
$$




\subsection{A two point estimate using Poincaré inequality}

For two measures $m, \nu$ on $(X, d)$, for $R>0, x \in X$, we define a 'truncated maximal function'

$$
M_{R}^{m} \nu(x)=\sup _{0<r<R} \frac{\nu(B(x, r))}{m(B(x, r))} .
$$

If $\nu \ll m$, then the above expression is the truncated maximal function of the RadonNikodym derivative $\frac{d \nu}{d m}$. However, in the lemma below $\nu$ will be the energy measure, and hence the measure $\nu$ and $m$ might be mutually singular. In the following lemma, by $\mathcal{C}(X)$ we mean the space of continuous functions on $X$.

Lemma 2.4. (see [HeKo98, Lemma 5.15]) Let $(X, d, m, \mathcal{E}, \mathcal{F})$ be an $M M D$ space that satisfies (VD), $\mathrm{PI}(\Psi)$, where $\Psi$ satisfies (1.3). There exists $C>0$ such that for all $x_{0} \in X, R>0, x, y \in B\left(x_{0}, C^{-1} R\right)$, and for all $u \in \mathcal{C}(X) \cap \mathcal{F}_{\text {loc }}$

$$
|u(x)-u(y)|^{2} \leq C \Psi(R)\left(M_{R}^{m} \Gamma(u, u)(x)+M_{R}^{m} \Gamma(u, u)(y)\right),
$$

where $\Gamma(u, u)$ denotes the energy measure of $u$.

Proof. The proof in [HeKo98] applies to our setting with minor modifications. For the convenience of the reader, we recall the proof below.

Let $\delta \in(0,1)$ be a constant that will be chosen later. For a ball $B$, by $u_{B}$ we denote $\frac{1}{m(B)} \int u d m$. Define $B_{x, i}=B\left(x, 2^{-i} \delta R\right)$ for $i \in\{0,1,2, \ldots\}$. We estimate

$$
\begin{aligned}
& \left|u(x)-u_{B_{x, 0}}\right| \leq \sum_{i=0}^{\infty}\left|u_{B_{x, i}}-u_{B_{x, i+1}}\right|=\sum_{i=0}^{\infty} \frac{1}{m\left(B_{x, i+1}\right)}\left|\int_{B_{x, i+1}}\left(u-u_{B_{x, i}}\right) d m\right| \\
& \leq \sum_{i=0}^{\infty} \frac{1}{m\left(B_{x, i+1}\right)} \int_{B_{x, i+1}}\left|u-u_{B_{x, i}}\right| d m \\
& \lesssim \frac{1}{m\left(B_{x, i}\right)} \int_{B_{x, i}}\left|u-u_{B_{x, i}}\right| d m \quad \text { (since } B_{x, i+1} \subset B_{x, i} \text { and by (VD)) } \\
& \leq \sum_{i=0}^{\infty}\left(\frac{1}{m\left(B_{x, i}\right)} \int_{B_{x, i}}\left|u-u_{B_{x, i}}\right|^{2} d m\right)^{1 / 2} \quad \text { (by Hölder inequality) } \\
& \lesssim \sum_{i=0}^{\infty} \Psi\left(2^{-i} \delta R\right)^{1 / 2}\left(\frac{1}{m\left(B_{x, i}\right)} \int_{B\left(x, A \delta 2^{-i} R\right)} d \Gamma(u, u)\right)^{1 / 2} \quad(\text { by } \operatorname{PI}(\Psi)) \\
& \lesssim \sum_{i=0}^{\infty} \Psi(R)^{1 / 2} 2^{-\beta_{1} i / 2}\left(M_{A \delta R}^{m} \Gamma(u, u)(x)\right)^{1 / 2} \quad \text { (by (1.3) and (VD)) } \\
& \lesssim\left(\Psi(R) M_{A \delta R}^{m} \Gamma(u, u)(x)\right)^{1 / 2} .
\end{aligned}
$$

Similarly, by setting $B_{y}=B\left(y, \delta^{\prime} R\right)$, we obtain

$$
\left|u(x)-u_{B_{y}}\right| \lesssim\left(\Psi(R) M_{A \delta^{\prime} R}^{m} \Gamma(u, u)(y)\right)^{1 / 2} .
$$


We choose $C>1$ large enough and $\delta^{\prime}>\delta$ so that $x, y \in B\left(x_{0}, C^{-1} R\right)$ implies $B_{x} \subset B_{y}$. For example, it suffices to choose $C, \delta, \delta^{\prime}$ so that

$$
2 C^{-1}+\delta<\delta^{\prime}
$$

We also require

$$
A \delta \leq A \delta^{\prime} \leq 1
$$

so that $M_{A \delta R}^{m} \Gamma(u, u)(x) \leq M_{R}^{m} \Gamma(u, u)(x)$ and $M_{A \delta R}^{m} \Gamma(u, u)(y) \leq M_{R}^{m} \Gamma(u, u)(y)$.

Evidently, given any $A>1$, it is possible to choose $C>1,0<\delta \leq \delta^{\prime}<1$ such that (2.8) and (2.9) are satisfied. We fix one such $C, \delta, \delta^{\prime}$ for the remainder of the proof.

By $B_{x, 0} \subset B_{y}, m\left(B_{y}\right) \lesssim m\left(B_{x, 0}\right),(\mathrm{VD})$ and $\mathrm{PI}(\Psi)$, we obtain

$$
\begin{aligned}
\left|u_{B_{x, 0}}-u_{B_{y}}\right| & \leq \frac{1}{m\left(B_{x, 0}\right)} \int_{B_{x, 0}}\left|u-u_{B_{y}}\right| d m \lesssim \frac{1}{m\left(B_{y}\right)} \int_{B_{y}}\left|u-u_{B_{y}}\right| d m \\
& \lesssim\left(\Psi(R) \frac{\Gamma(u, u)\left(B\left(y, A \delta^{\prime} R\right)\right)}{m\left(B_{y}\right)}\right)^{1 / 2} \leq\left(\Psi(R) M_{A \delta^{\prime} R}^{m} \Gamma(u, u)(y)\right)^{1 / 2}
\end{aligned}
$$

Combining (2.6), (2.7), (2.10), $A \delta \leq A \delta^{\prime} \leq 1$, we obtain the desired bound

$$
\begin{aligned}
|u(x)-u(y)| & \leq\left|u(x)-u_{B_{x, 0}}\right|+\left|u_{B_{x}, 0}-u_{B_{y}}\right|+\left|u(y)-u_{B_{y}}\right| \\
& \lesssim \Psi(R)^{1 / 2}\left(M_{R}^{m} \Gamma(u, u)(x)+M_{R}^{m} \Gamma(u, u)(y)\right)^{1 / 2} .
\end{aligned}
$$

The telescoping sum argument has been applied in the context of anomalous diffusions previously in [BCK, p. 1654]. However, in [BCK] the argument is used in the 'strongly recurrent case'. One of the main novelties of this work is to extract useful estimates from that argument without further assumptions on volume growth.

\section{$2.3 \quad$ A partition of unity}

The use of partition of unity with functions of small Dirichlet energy indexed by a net is well-known [Ka86, p. 235], [BBK, p. 504]. Since we do not have a reference to give for the requirement in (c) below, we provide the details.

Lemma 2.5. Let $(X, d, m, \mathcal{E}, \mathcal{F})$ be an $M M D$ space that satisfies $(\mathrm{VD})$, and $\operatorname{Cap}(\Psi)_{\leq}$. Let $\varepsilon>0$ and let $V$ denote any $\varepsilon$-net. Let $\varepsilon<\operatorname{diam}(X, d) / A_{2}$, where $A_{2} \geq 1$ is the constant in $\operatorname{Cap}(\Psi)_{\leq}$. Then, there exists a family of functions $\left\{\psi_{z}: z \in V\right\}$ that satisfies the following properties:

(a) $\left\{\psi_{z}: z \in V\right\}$ is partition of unity $\sum_{z \in V} \psi_{z} \equiv 1$.

(b) $\psi_{z} \in C_{c}(X) \cap \mathcal{F}$ with $0 \leq \psi_{z} \leq 1,\left.\psi_{z}\right|_{B(z, \varepsilon / 4)} \equiv 1$, and $\left.\psi_{z}\right|_{B(z, 5 \varepsilon / 4)^{c}} \equiv 0$.

(c) For all $z \in V, z^{\prime} \in V \backslash\{z\}$, we have $\left.\psi_{z^{\prime}}\right|_{B(z, \varepsilon / 4)} \equiv 0$. (this follows from (a) and (b)). 
(d) There exists $C>1$ such that for all $z \in V$,

$$
\mathcal{E}\left(\psi_{z}, \psi_{z}\right) \leq C \frac{m(B(z, \varepsilon))}{\Psi(\varepsilon)} .
$$

Proof. For $z \in V$, we define the corresponding 'Voronoi cell' $R_{z}$ as

$$
R_{z}=\left\{p \in X: d(p, z)=d(p, V)=\min _{v \in V} d(p, v)\right\} .
$$

We denote its $\varepsilon / 4$-neighbourhood by $R_{z}^{\varepsilon / 4}=\cup_{x \in R_{z}} B(x, \varepsilon / 4)$. By the triangle inequality, we have

$$
B(z, \varepsilon / 2) \subset R_{z} \subset \bar{B}(z, \varepsilon), \quad \bigcup_{z \in V} R_{z}=X,
$$

and

$$
p \in B(z, \varepsilon / 2) \text { implies that } p \notin V_{w} \text { for } w \in V \backslash\{z\} .
$$

By (2.12), and the triangle inequality, we have

$$
v, w \in V \text { and } v \neq w \text {, imply that } B(z, \varepsilon / 4) \cap R_{w}^{\varepsilon / 4}=\emptyset .
$$

For $z \in V$, let $N_{z}$ denote an $\varepsilon /\left(4 A_{1}\right)$-net of $R_{z}$, where $A_{1}$ denote the constant in $\operatorname{cap}(\Psi)_{\leq}$. For each $w \in N_{z}$, by $\operatorname{cap}(\Psi)_{\leq},(1.3)$, (VD), there exists $C_{1}>1$ such that for $w \in N_{z}, z \in$ $V$, we have a non-negative function $\rho_{w} \in C_{c}(X) \cap \mathcal{F}$ that satisfies

$$
\left.\rho_{w}\right|_{B\left(w, \mathcal{E} /\left(4 A_{1}\right)\right.} \equiv 1,\left.\quad \rho_{w}\right|_{B(w, \varepsilon / 4)^{c}} \equiv 0, \quad \mathcal{E}\left(\rho_{w}, \rho_{w}\right) \leq C_{1} \frac{m(B(w, \varepsilon)}{\Psi(\varepsilon)} .
$$

Hence by (VD) and (2.14), we obtain a family of functions $\left\{\phi_{z}: z \in V\right\}$ that satisfy

$$
\phi_{z}=\max _{w \in N_{z}} \rho_{w} \text { such that }\left.\phi_{w}\right|_{R_{z}} \equiv 1,\left.\phi_{w}\right|_{\left(R_{z}^{\varepsilon / 4}\right)^{c}} \equiv 0, \mathcal{E}\left(\phi_{z}, \phi_{z}\right) \lesssim \frac{m(B(z, \varepsilon)}{\Psi(\varepsilon)} \text {. }
$$

Define

$$
\psi_{z}:=\frac{\phi_{z}}{\sum_{w \in V} \phi_{w}} .
$$

Property (a) is immediate. Properties (b) and (c) follow from (2.15), (2.13), and (2.11). Property (d) follows from (2.15), (VD), Leibniz rule, chain rule and Cauchy-Schwarz inequality, we obtain

$$
\begin{aligned}
\mathcal{E}\left(\psi_{z}, \psi_{z}\right) & \lesssim \sup \left(\sum_{w \in V} \phi_{w}\right)^{-2}\left(\mathcal{E}\left(\phi_{z}, \phi_{z}\right)+\left(\sup \phi_{z}\right)^{2} \sum_{w \in V \cap B(z, 5 \varepsilon / 2)} \mathcal{E}\left(\phi_{w}, \phi_{w}\right)\right) \\
& \lesssim \sum_{w \in V \cap B(z, 5 \varepsilon / 2)} \frac{m(B(w, \varepsilon))}{\Psi(\varepsilon)} \lesssim \frac{m(B(z, \varepsilon))}{\Psi(\varepsilon)}
\end{aligned}
$$




\subsection{Proof of the main result}

We recall an elementary lemma from [GT12].

Lemma 2.6. ([GT12, Lemma 6.3]) Let $(X, d)$ be a metric space. Define $N_{\varepsilon}(x, y)$ as the minimal value of $N$ such that there exists an $\varepsilon$-chain $\left\{x_{i}\right\}_{i=0}^{N}$ between $x$ and $y$. If $d_{\varepsilon}(x, y)<\infty$ for some $x, y \in X, \varepsilon>0$, then

$$
\left\lceil\frac{d_{\varepsilon}(x, y)}{\varepsilon}\right\rceil \leq N_{\varepsilon}(x, y) \leq 9\left\lceil\frac{d_{\varepsilon}(x, y)}{\varepsilon}\right\rceil .
$$

Proof of Theorem 1.6. Let $A_{2}$ denote the constant in Cap $(\Psi)_{\leq}$. Since $d_{\varepsilon} \leq d_{\varepsilon^{\prime}}$ whenever $\varepsilon^{\prime} \leq \varepsilon$, by replacing $\varepsilon$ by $\varepsilon /\left(2 A_{2}\right)$ if necessary and by using (1.3), we assume that $\varepsilon<$ $\operatorname{diam}(X, d) / A_{2}$.

Fix $x, y \in X, \varepsilon>0$ such that $d(x, y) \geq \varepsilon$. Set $\varepsilon^{\prime}=\varepsilon / 3$. Let $V$ be an $\varepsilon^{\prime}$-net such that $\{x, y\} \subset V$. Define $\hat{u}: V \rightarrow[0, \infty)$ as

$$
\hat{u}(z):=N_{\varepsilon}(x, z),
$$

where $N_{\varepsilon}(x, z)$ is as defined in Lemma 2.6. By Lemma 2.2, $\hat{u}$ is finite. By definition,

$$
\left|\hat{u}\left(z_{1}\right)-\hat{u}\left(z_{2}\right)\right| \leq 1 \quad \text { for all } z_{1}, z_{2} \in V \text { such that } d\left(z_{1}, z_{2}\right)<\varepsilon \text {. }
$$

Let $\left\{\psi_{z}: z \in V\right\}$ denote the partition of unity defined in Lemma 2.5. Define $u: X \rightarrow$ $[0, \infty)$ as

$$
u(p):=\sum_{z \in V} \hat{u}(z) \psi_{z}(p) .
$$

For any ball $B\left(x_{0}, r\right), x_{0} \in X, r>0$, by Lemma 2.5 we have

$$
u(p)=\sum_{z \in V \cap B\left(x_{0}, r+5 \varepsilon^{\prime} / 4\right)} \hat{u}(z) \psi_{z}(p) \quad \text { for all } p \in B\left(x_{0}, r\right) .
$$

Since $V \cap B\left(x_{0}, r+5 \varepsilon^{\prime} / 4\right)$ is a finite set by (VD), we obtain that $u \in \mathcal{F}_{\text {loc. By Lemma }}$ 2.5(b), we have $\left.u\right|_{B\left(z, \varepsilon^{\prime} / 4\right)} \equiv \hat{u}(z)$ for all $z \in V$. Therefore by [CF, Theorem 4.3.8], the push-forward measure of $\Gamma(u, u)$ by $u$ is absolutely continuous with respect to the 1-dimensional Lebesgue measure. Therefore, we obtain

$$
\Gamma(u, u)\left(B\left(z, \varepsilon^{\prime} / 4\right)\right)=0 \quad \text { for all } z \in V .
$$

By (2.18) and Lemma 2.5(a), we have

$$
u(p)=\hat{u}(z)+\sum_{w \in V \cap B\left(z, 9 \varepsilon^{\prime} / 4\right)}(\hat{u}(w)-\hat{u}(z)) \psi_{w}(p) \quad \text { for all } p \in B\left(z, \varepsilon^{\prime}\right), z \in V .
$$

By (VD), there exits $C_{1}>1$ such that $\sup _{z \in V}\left|V \cap B\left(z, 9 \varepsilon^{\prime} / 4\right)\right| \leq C_{1}$. By (2.20), and Cauchy-Schwarz inequality, there exists $C_{2}>1$ such that the following holds: for all 
$z \in V$, we have

$$
\begin{aligned}
\Gamma(u, u)\left(B\left(z, \varepsilon^{\prime}\right)\right) & \leq C_{1} \sum_{w \in V \cap B\left(z, 9 \varepsilon^{\prime} / 4\right)}(\hat{u}(w)-\hat{u}(z))^{2} \mathcal{E}\left(\psi_{w}, \psi_{w}\right) \\
& \left.\lesssim \sum_{w \in V \cap B\left(z, 9 \varepsilon^{\prime} / 4\right)} \frac{m\left(B\left(w, \varepsilon^{\prime}\right)\right)}{\Psi\left(\varepsilon^{\prime}\right)} \quad \text { (by }(2.17) \text { and Lemma } 2.5(\mathrm{~d})\right) \\
& \left.\leq C_{2} \frac{m\left(B\left(z, \varepsilon^{\prime} / 2\right)\right)}{\Psi(\varepsilon)} \quad \text { (by (VD) and }(1.3)\right)
\end{aligned}
$$

By Lemma 2.5, (2.21) and (VD), there exists $C_{3}>0$ such that for all $z \in X, r \geq \varepsilon^{\prime} / 4$, we have

$$
\Gamma(u, u)(B(z, r)) \leq C_{2} \sum_{w \in B\left(z, r+5 \varepsilon^{\prime} / 4\right)} \frac{m\left(B\left(w, \varepsilon^{\prime} / 2\right)\right)}{\Psi(\varepsilon)} \leq C_{3} \frac{m(B(z, r))}{\Psi(\varepsilon)}
$$

Combining (2.19) and (2.22), we obtain

$$
M_{R}^{m} \Gamma(u, u)(z)=\sup _{r<R} \frac{\Gamma(u, u)(B(z, r))}{m(B(z, r))} \leq \frac{C_{3}}{\Psi(\varepsilon)} \quad \text { for all } z \in V, R>0 .
$$

By (2.23), Lemma 2.4, $\hat{u}(x)=0, \hat{u}(y)=N_{\varepsilon}(x, y)$, and (1.3), there exists $C_{4}>0$ such that

$$
N_{\varepsilon}(x, y)^{2} \leq C_{4} \frac{\Psi(d(x, y))}{\Psi(\varepsilon)} \text { for all } x, y \in X, \varepsilon \leq d(x, y)
$$

Combining the above estimate along with Lemma 2.6, we obtain (1.4). We obtain (1.5) using (1.4) and $\lim _{\varepsilon \downarrow 0} \Psi(\varepsilon)=0$.

Remark 2.7. (a) The constant $C$ in (1.4) can be chosen to depend only on the constants associated with the assumptions $(\mathrm{VD}), \mathrm{PI}(\Psi), \operatorname{cap}(\Psi) \leq$ and (1.3).

(b) The proof of the estimate on $d_{\varepsilon}(x, y)$ uses $\operatorname{cap}(\Psi)_{\leq}$only at scales less than $\varepsilon$, whereas it relies on $\mathrm{PI}(\Psi)$ for scales up to the order of $d(x, y)$. In other words, our argument relies on the Poincaré inequality on a larger range of scales than it relies on the capacity upper bound.

Proof of Corollary 1.10. Let $0<s<r<\operatorname{diam}(X, d)$. By uniform perfectness of $(X, d)$ (more precisely, by (2.4)), there exists $x, x_{r} \in X$ such that $r / 2 \leq d\left(x, x_{r}\right)<r$.

By (1.3), it suffices to consider the case $s \leq r / 2$. Therefore,

$$
\begin{aligned}
\frac{\Psi(r)}{\Psi(s)} & \asymp \frac{\Psi\left(d\left(x, x_{r}\right)\right)}{\Psi(s)} \quad(\text { by }(1.3)) \\
& \gtrsim\left(\frac{d\left(x, x_{r}\right)}{s}\right)^{2} \gtrsim\left(\frac{r}{s}\right)^{2} \quad(\text { by }(1.4)) .
\end{aligned}
$$




\subsection{Application to heat kernel estimates}

Definition 2.8. Let $U \subset V$ be open sets in $X$ with $U \subset \bar{U} \subset V$. We say a continuous function $\phi$ is a cutoff function for $U \subset V$ if $\phi=1$ on a neighbourhood of $\bar{U}$ and $\operatorname{supp}(\phi) \subset$ $V$.

We recall the cutoff Sobolev inequality $\operatorname{CS}(\Psi)$ for an $\operatorname{MMD} \operatorname{space}(X, d, m, \mathcal{E}, \mathcal{F})$ : there exists $C_{S}>0$ such that for any $x \in X, R, r>0$, there exists a cutoff function $\phi$ for $B(x, R) \subset B(x, R+r)$ such that

$$
\int_{B(x, R+r) \backslash B(x, R)} f^{2} d \Gamma(\phi, \phi) \leq \frac{1}{8} \int_{B(x, R+r) \backslash B(x, R)} \phi^{2} d \Gamma(f, f)+\frac{C_{S}}{\Psi(r)} \int_{B(x, R+r) \backslash B(x, R)} f^{2} d m,
$$

for all $f \in \mathcal{F}$.

For $\Psi$ satisfying (1.3), we define

$$
\Phi(s)=\sup _{r>0}\left(\frac{s}{r}-\frac{1}{\Psi(r)}\right) .
$$

Corollary 2.9. Let $(X, d, m, \mathcal{E}, \mathcal{F})$ be an $M M D$ space that satisfies $(\mathrm{VD}), \operatorname{PI}(\Psi)$, and $\mathrm{CS}(\Psi)$, where $\Psi$ satisfies (1.3). Then we have matching upper and lower bounds on the heat kernel as given in

$$
p_{t}(x, y) \asymp \frac{C}{V\left(x, \Psi^{-1}(t)\right)} \exp \left(-c t \Phi\left(\frac{d_{\varepsilon}(x, y)}{t}\right)\right),
$$

where $\Phi$ is as defined in (2.24), and $\varepsilon=\varepsilon(t, x, y)$ is chosen so that

$$
\varepsilon(t, x, y)=\sup \left\{\varepsilon>0: \frac{\Psi(\varepsilon)}{\varepsilon} d_{\varepsilon}(x, y) \leq t\right\} .
$$

Here $\asymp$ in (2.25) means that both $\leq$ and $\geq$ are true, but the positive constants $C$ and $c$ may be different for upper and lower bounds.

Proof. This follows from [GHL, Theorem 1.2], [GT12, Theorem 6.5] and Theorem 1.6.

Lemma 2.10. Let $\Psi:(0, \infty) \rightarrow(0, \infty)$ be a continuous, increasing bijection such that

$$
C_{1}^{-1}\left(\frac{R}{r}\right)^{\beta_{1}} \leq \frac{\Psi(R)}{\Psi(r)} \leq C_{1}\left(\frac{R}{r}\right)^{\beta_{2}},
$$

for some constants $1<\beta_{1} \leq \beta_{2}$ and $C_{1}>1$. Let $\Phi$ be the function defined in (2.24). Then, there exists $C_{2}>0$ such that

$$
C_{2}^{-1}\left(\frac{S}{s}\right)^{\beta_{2} /\left(\beta_{2}-1\right)} \leq \frac{\Phi(S)}{\Phi(s)} \leq C_{2}\left(\frac{S}{s}\right)^{\beta_{1} /\left(\beta_{1}-1\right)}, \text { for all } 0<s \leq S .
$$


Proof. For $\lambda \geq 1, r>0$, we have

$$
\begin{aligned}
\Phi(s) & =\sup _{r>0}\left(\frac{s}{r}-\frac{1}{\Psi(r)}\right)=\sup _{r>0}\left(\frac{s}{r \lambda}-\frac{1}{\Psi(r \lambda)}\right) \\
& \leq \sup _{r>0}\left(\frac{s}{r \lambda}-\frac{1}{C_{1} \lambda^{\beta_{2}} \Psi(r)}\right) \quad(\text { by }(2.26)) \\
& \leq \frac{1}{C_{1} \lambda^{\beta_{2}}} \sup _{r>0}\left(\frac{s C_{1}^{-1} \lambda^{\beta_{2}-1}}{r}-\frac{1}{\Psi(r)}\right) \\
& \leq \frac{1}{C_{1} \lambda^{\beta_{2}}} \Phi\left(s C_{1}^{-1} \lambda^{\beta_{2}-1}\right) .
\end{aligned}
$$

By choosing $S / s=C_{1}^{-1} \lambda^{\beta_{2}-1}$ in the above estimate, we obtain the lower bound on $\frac{\Phi(S)}{\Phi(s)}$ in (2.27). The proof of upper bound in (2.27) is analogous.

We prove the following generalization of Corollary 1.8.

Theorem 2.11. Let $(X, d, m, \mathcal{E}, \mathcal{F})$ be an $M M D$ space. Let $\Psi:(0, \infty) \rightarrow(0, \infty)$ be an increasing bijection that satisfies (2.26). Assume that $(X, d, m, \mathcal{E}, \mathcal{F})$ satisfies the following estimate on its heat kernel $p_{t}(\cdot, \cdot)$ :

$$
p_{t}(x, y) \asymp \frac{C}{V\left(x, \Psi^{-1}(t)\right)} \exp \left(-c t \Phi\left(\frac{d(x, y)}{t}\right)\right),
$$

for all $x, y \in X, t>0$, where $V(x, r)=m(B(x, r))$, where $\Phi:(0, \infty) \rightarrow(0, \infty)$ be as defined in (2.24). Here $\asymp$ in (2.28) means that both $\leq$ and $\geq$ are true, but the positive constants $C$ and $c$ may be different for upper and lower bounds. Then the metric $d$ satisfies the chain condition: there exists $K>1$ such that

$$
d(x, y) \leq d_{\varepsilon}(x, y) \leq K d(x, y) \quad \text { for all } \varepsilon>0 \text { and for all } x, y \in X .
$$

Proof. By [GT12, Lemma 6.4] and (2.26), $\varepsilon=\varepsilon(t, x, y)$ satisfies

$$
\varepsilon^{\beta_{2}-1} d_{\varepsilon}(x, y) \lesssim \frac{\Psi(\varepsilon)}{\varepsilon} d_{\varepsilon}(x, y)=t .
$$

Note that

$$
\begin{aligned}
t \Phi\left(\frac{2 \Psi^{-1}(t)}{t}\right) & =\sup _{r>0}\left(\frac{2 \Psi^{-1}(t)}{r}-\frac{t}{\Psi(r)}\right) \\
& \leq \sup _{r>0}\left(\frac{2 \Psi^{-1}(t)}{r}-C_{1}^{-1} \min \left(\frac{\Psi^{-1}(t)^{\beta_{1}}}{r^{\beta_{1}}}, \frac{\Psi^{-1}(t)^{\beta_{2}}}{r^{\beta_{2}}}\right)\right) \\
& \lesssim 1
\end{aligned}
$$

For $x \in X, r>0$, by integrating the lower bound in (1.6), over the ball $B(x, 2 r)$ with $\Psi^{-1}(t)=r$ and using (2.30), we obtain

$$
\frac{V(x, 2 r)}{V(x, r)} \lesssim \int_{B(x, 2 r)} p_{t}(x, y) m(d y) \leq 1,
$$


which implies the volume doubling property. By the argument in the proof of Theorem 1.2 in [GHL, proof of (8.6)], the MMD space $(X, d, m, \mathcal{E}, \mathcal{F})$ satisfies $\mathrm{PI}(\Psi)$. By $(2.4)$ and [Hei, Exercise 13.1], $(X, d, m)$ satisfies the reverse volume doubling property; that is, there exist $c, \alpha>0$ such that

$$
\frac{V(x, R)}{V(x, r)} \geq c^{-1}\left(\frac{R}{r}\right)^{\alpha}, \quad \text { for all } 0<r<R<\operatorname{diam}(X, d) .
$$

By [GHL, Theorem 1.2], the MMD space $(X, d, m, \mathcal{E}, \mathcal{F})$ satisfies $(\mathrm{VD}), \mathrm{PI}(\Psi)$, and $\mathrm{CS}(\Psi)$. In [GHL, Theorem 1.2] assumes that the space is unbounded, the same result also extends to the bounded case [Lie, Theorem 3.2]; see [KM, Remark 2.9] for further discussion. Therefore by Corollary 2.9 and (2.28), for all $x, y \in X$ and $t>0$, we have

$$
\begin{aligned}
c_{2} \exp \left(-C_{2} t \Phi\left(\frac{d(x, y)}{t}\right)\right) & \leq V\left(x, \Psi^{-1}(t)\right) p_{t}(x, y) \\
& \leq C_{1} \exp \left(-c_{1} t \Phi\left(\frac{d_{\varepsilon}(x, y)}{t}\right)\right)
\end{aligned}
$$

where $\varepsilon=\varepsilon(t, x, y)$. Therefore for $x, y \in X, t>0$

$$
c_{1} t \Phi\left(\frac{d_{\varepsilon}(x, y)}{t}\right) \leq C_{2} t \Phi\left(\frac{d(x, y)}{t}\right)+\log \left(C_{1} / c_{2}\right)
$$

Hence, by [GT12, Lemma 3.19], for every $x, y \in X$, there exists $t_{x, y}>0$ such that for all $0<t<t_{x, y}$, we have

$$
c_{1} t \Phi\left(\frac{d_{\varepsilon}(x, y)}{t}\right) \leq 2 C_{2} t \Phi\left(\frac{d(x, y)}{t}\right)
$$

By the above estimate and Lemma 2.10

$$
d_{\varepsilon}(x, y) \lesssim d(x, y), \quad \text { for all } x, y>0, t<t_{x, y} \text {, where } \varepsilon=\varepsilon(t, x, y)
$$

By (2.29) and $d_{\varepsilon} \geq d$, we obtain

$$
\varepsilon(t, x, y) \lesssim\left(\frac{t}{d(x, y)}\right)^{1 /\left(\beta_{2}-1\right)}
$$

Hence $\lim _{t \rightarrow 0} \varepsilon(t, x, y)=0$. Since $\varepsilon \mapsto d_{\varepsilon}(x, y)$ is non-increasing, and $\lim _{t \downarrow 0} \varepsilon(t, x, y)=0$, by letting $t \downarrow 0$ in (2.31), we obtain

$$
\lim _{\varepsilon \downarrow 0} d_{\varepsilon}(x, y) \lesssim d(x, y), \quad \text { for all } x, y \in X .
$$

Hence for all $x, y \in X, \varepsilon>0$, we obtain $d_{\varepsilon}(x, y) \asymp d(x, y)$.

Proof of Corollary 1.8. This is a special case of Theorem 2.11 with $\Psi(r)=r^{\beta}$. 
Remark 2.12. Let $\Psi: X \times[0, \infty) \rightarrow[0, \infty)$ is a regular scale function in the sense of [BM, Definition 5.4]; that is, there exists $C>1, \beta_{1}, \beta_{2}>0$ such that for all $x, y \in X, 0<s \leq r$, we have

$$
C^{-1}\left(\frac{r}{d(x, y) \vee r}\right)^{\beta_{2}}\left(\frac{d(x, y) \vee r}{s}\right)^{\beta_{1}} \leq \frac{\Psi(x, r)}{\Psi(y, s)} \leq C\left(\frac{r}{d(x, y) \vee r}\right)^{\beta_{1}}\left(\frac{d(x, y) \vee r}{s}\right)^{\beta_{2}} .
$$

Further, assume that the MMD space $(X, d, m, \mathcal{E}, \mathcal{F})$ satisfies $(\mathrm{VD}), \operatorname{PI}(\Psi)$, and $\mathrm{CS}(\Psi)$. In this case, we can obtain sharp heat kernel bounds as follows: by a change of metric as done in [BM, Proposition 5.7], we can reduce it to the case $\Psi(r)=r^{\beta}$, which can be handled using Corollary 2.9.

\section{Acknowledgements}

I thank Takashi Kumagai for inspiring this work by asking if Corollary 1.8 could be true. It is a pleasure to acknowledge Naotaka Kajino and Takashi Kumagai for helpful references and discussions. This work was carried out at the Research Institute for Mathematical Sciences, Kyoto University and at Kobe University. I am grateful to Takashi Kumagai, Naotaka Kajino, Jun Kigami, and Ryoki Fukushima for support and hospitality during the visit. The author thanks the anonymous referee for a careful reading of the paper and helpful suggestions.

\section{References}

[Ar67] D. G. Aronson. Bounds for the fundamental solution of a parabolic equation. Bull. Amer. Math. Soc. 731967 890-896. MR0217444

[AS67] D. G. Aronson, J. Serrin. Local behavior of solutions of quasilinear parabolic equations. Arch. Rational Mech. Anal. 251967 81-122. MR0244638

[Bar98] M. T. Barlow, Diffusions on fractals, Lecture Notes in Math. 1690, 1-121, Springer, Berlin, 1998. MR1668115

[BBK] M.T. Barlow, R.F. Bass and T. Kumagai. Stability of parabolic Harnack inequalities on metric measure spaces. J. Math. Soc. Japan (2) 58 (2006), 485-519. MR2228569

[BCK] M. T. Barlow, T. Coulhon, T. Kumagai. Characterization of sub-Gaussian heat kernel estimates on strongly recurrent graphs, Comm. Pure Appl. Math. 58 (2005), no. 12, 1642-1677. MR2177164

[BM] M.T. Barlow, M. Murugan. Stability of the elliptic Harnack inequality, Ann. of Math. (2) 187 (2018), 777-823. MR3779958

[BBI] D. Burago, Y. Burago and S. Ivanov. A course in Metric Geometry, Graduate Studies in Mathematics, 33. American Mathematical Society, Providence, RI, 2001. MR1835418 
[CF] Z.-Q. Chen and M. Fukushima, Symmetric Markov Processes, Time Change, and Boundary Theory, London Mathematical Society Monographs Series 35, Princeton University Press, Princeton, NJ, 2012. MR2849840

[CS] T. Coulhon, L. Saloff-Coste. Variétés riemanniennes isométriques à l'infini, Rev. Mat. Iberoamericana 11 (1995), no. 3, 687-726. MR1363211

[FOT] M. Fukushima, Y. Oshima, and M. Takeda, Dirichlet Forms and Symmetric Markov Processes, 2nd ed., de Gruyter, Berlin, 2011. MR2778606

[GHL] A. Grigor'yan, J. Hu, K.-S. Lau. Generalized capacity, Harnack inequality and heat kernels of Dirichlet forms on metric spaces. J. Math. Soc. Japan 67 1485-1549 (2015). MR3417504

[GT12] A. Grigor'yan, A. Telcs. Two-sided estimates of heat kernels on metric measure spaces. Ann. Probab. 40 (2012), no. 3, 1212-1284. MR2962091

[HaKu99] B. M. Hambly, T. Kumagai. Transition density estimates for diffusion processes on post critically finite self-similar fractals. Proc. London Math. Soc. (3) 78 (1999), no. 2, 431-458. MR1665249

[HaKu04] B. M. Hambly, T. Kumagai, Heat kernel estimates for symmetric random walks on a class of fractal graphs and stability under rough isometries. Fractal geometry and applications: a jubilee of Benoît Mandelbrot, Part 2, 233-259, Proc. Sympos. Pure Math., 72, Part 2, Amer. Math. Soc., Providence, RI, 2004. MR2112125

[Hei] J. Heinonen, Lectures on Analysis on Metric Spaces, Universitext, Springer-Verlag, New York, 2001, x+140 pp. MR1800917

[HeKo98] J. Heinonen, P. Koskela. Quasiconformal maps in metric spaces with controlled geometry, Acta Math. 181 (1998), no. 1, 1-61. MR1654771

[HR] M. Hino, J. A. Ramirez, Small-time Gaussian behavior of symmetric diffusion semigroups. Ann. Probab. 31 (2003), no. 3, 1254-1295. MR1988472

[KM] N. Kajino, M. Murugan. On singularity of energy measures for symmetric diffusions with full off-diagonal heat kernel estimates (preprint) 2020.

[Ka86] M. Kanai. Rough isometries and the parabolicity of Riemannian manifolds. J. Math. Soc. Japan 38 (1986), no. 2, 227-238. MR0792983

[Lie] J. Lierl, Scale-invariant boundary Harnack principle on inner uniform domains in fractal-type spaces, Potential Anal. 43 (2015), no. 4, 717-747. MR3432457

[MT] J. M. Mackay, J. T. Tyson. Conformal dimension. Theory and application. University Lecture Series, 54. American Mathematical Society, Providence, RI, 2010.

Department of Mathematics, University of British Columbia, Vancouver, BC V6T 1Z2, Canada.

mathav@math.ubc.ca 